\title{
The stellar content of OMC 2/3
}

\author{
M. Nielbock ${ }^{1,2}$, R. Chini ${ }^{1}$, and S. A. H. Müller ${ }^{1}$ \\ 1 Astronomisches Institut der Ruhr-Universität Bochum, Universitätsstrasse 159/NA 7, 44780 Bochum, Germany \\ 2 SEST, European Southern Observatory, Alonso de Cordova 3107, Santiago, Chile
}

Received 25 April 2003 / Accepted 17 June 2003

\begin{abstract}
We have revisited the stellar content of OMC 2 and OMC 3 by means of MIR imaging and NIR photometry; in addition, we have extended the existing (sub)mm maps by a huge $1200 \mu \mathrm{m}$ map obtained with SIMBA showing new sources and filamentary features for the first time at that wavelength. The MIR data reveal 43 new sources at $N$ and $Q$ which are partly associated with dense condensations at millimetre wavelengths. Six close binary sources could be resolved at locations where existing (sub)mm maps only show single emission peaks; three of them are classified as early (B-type) systems, one of them is compatible with type $\mathrm{K}$ while the remaining two seem to be T Tauri stars. Furthermore, the MIR images indicate the existence of separate circumstellar discs in the K-binary system. NIR colour-colour and colour-magnitude diagrams obtained from 2MASS data are examined to explore the physical properties of the sources and to derive the distribution of $J$ luminosities. There is a clear decrease in luminosity and thus in stellar mass when going from South to North. Likewise, there is an anti-correlation between Class I and II objects in both regions: while OMC 2 contains twice as much Class II objects compared to Class I, the situation is reversed in $\mathrm{OMC} 3$.
\end{abstract}

Key words. ISM: dust - stars: circumstellar matter, formation

\section{Introduction}

The GMC complex Orion A is usually separated into three distinct components, i.e. OMC 1,2 and 3 . The OMC $2 / 3$ region has turned out to be one of the most active sites of ongoing low and intermediate-mass star formation known today. Numerous observations at optical, NIR, FIR, sub-mm and $\mathrm{cm}$ wavelengths have been devoted to the stellar content of this region, and have revealed a young association with sources of different evolutionary stages.

Gatley et al. (1974) originally discovered OMC 2 as a star forming region by IR and $\mathrm{CO}$ measurements. They found 5 embedded IR sources within a cloud core of $\sim 1000 M_{\odot}$. This region is a small fraction of the cloud complex Orion A found by Kutner et al. (1977). A spatially well resolved study in the ${ }^{13} \mathrm{CO}$ line revealed a filamentary structure (Bally et al. 1987) of the entire region. Infrared studies (e.g. Rayner et al. 1989; Johnson et al. 1990; Jones et al. 1994; Ali \& DePoy 1995) discovered a population of young stars, many of them surrounded by circumstellar discs (e.g. O'Dell et al. 1993; McCaughrean \& O'Dell 1996; Hillenbrand et al. 1998).

During the first $1300 \mu \mathrm{m}$ continuum mapping of OMC 1 and 2, Mezger et al. (1990) found 10 compact sources. Subsequently, Chini et al. (1997) investigated OMC 2 and 3 at $1300 \mu \mathrm{m}$ with a better spatial coverage and higher sensitivity, and discovered a long integral shaped narrow dust ridge

\footnotetext{
Send offprint requests to: M. Nielbock, e-mail: mnielboc@eso.org

* Based on observations collected at the European Southern Observatory, La Silla, Chile.
}

ranging from $\mathrm{OMC} 1$ to $\mathrm{OMC} 3$ that contains 21 compact sources. Two of them were also detected by IRAS. $350 \mu \mathrm{m}$ imaging by Lis et al. (1998) suggested the presence of even 33 dust condensations within the area. Finally, the largest $1200 \mu \mathrm{m}$ continuum map of Orion A (Nyman et al. 2001) extends over $1^{\circ}$ in declination and depicts the total dust emission from that region. Many of the (sub)mm objects exhibit molecular $\mathrm{H}_{2}$ outflows (Castets \& Langer 1995; Yu et al. 1997).

A VLA survey by Reipurth et al. (1999) led to the detection of a number of radio sources, 11 of which were associated with previously detected (sub)mm condensations. Tsuboi et al. (2001) report on X-ray sources in OMC 3 and associate them with very young stellar objects.

Despite this wealth of data, the evolutionary stage of many objects is still unclear; this is true for the pure (sub)mm sources as well as for some NIR sources with (sub)mm and radio counterparts. A major reason for the uncertainty is the relatively large positional offset between data at different wavelengths which makes a unique correlation sometimes impossible. Another difficulty arises from the classification of the objects in the absence of sufficient spectral coverage - particularly at shorter wavelengths. As suggested by André et al. (1993), the ratio of bolometric to sub-mm luminosity may give an indication of the youth of a protostar; they argue that the bolometric luminosity is a measure of the protostellar mass, while the sub-mm luminosity represents the mass of the cold, circumstellar matter. The ratio of both quantities should therefore reflect the evolutionary stage of the protostellar system in the sense that the sub-mm luminosity must decrease with 
the ongoing accretion of the surrounding material. However, in practice the ratio $L_{\mathrm{bol}} / L_{\mathrm{smm}}$ is difficult - in most cases even impossible - to determine, because these earliest stellar stages evolve deeply within dust clouds so that NIR observations if available at all - suffer from heavy extinction. In addition, satellite (or airborne) FIR observations of protostars have been limited so far by sensitivity and spatial resolution so that $L_{\text {bol }}$ remains a poorly constrained quantity in most cases. Thus, it seems that further classification criteria for protostellar sources would be highly desirable.

The advent of sensitive ground-based MIR imaging devices is likely to improve the situation because they i) provide a sub-arcsec resolution, ii) have a better sensitivity than IRAS, and iii) are barely influenced by interstellar extinction. In addition, they fill an important gap in existing spectral energy distributions (SEDs) - right between the NIR and (sub)mm regimes, and thus seem to be ideally suited to elucidate the evolutionary stage of protostellar candidates.

In the present paper, we report on an $N$ and $Q$ band survey of OMC $2 / 3$ with TIMMI 2 following up our previous investigations of the youngest stars in Orion. Data from our new extended $1200 \mu \mathrm{m}$ continuum survey of the region with SIMBA as well as NIR data from the 2MASS database are also included.

\section{Observations}

The mid-infrared (MIR) observations were carried out in January 2001 and 2002 with TIMMI 2 (Dietzsch \& Reimann 1998; Reimann et al. 1998) at the ESO $3.6 \mathrm{~m}$ telescope, La Silla, Chile. TIMMI2 is a mid-infrared spectrograph and imager operated in the $M(5 \mu \mathrm{m})$, $N(10 \mu \mathrm{m})$ and $Q(20 \mu \mathrm{m})$ atmospheric bandpasses (cf. http://www. ls.eso.org/lasilla/Telescopes/360cat/ timmi/). We observed in the $N$ - and $Q$-bands. The first run was carried out using a set of filters centred at wavelengths of $11.9(N)$ and $17.8 \mu \mathrm{m}(Q)$. The $N$-band filter was replaced with one centred at $10.4 \mu \mathrm{m}$ for the second campaign, in which we re-measured nearly all the previous detections. The average seeing was about 0.77 being close to diffraction limited imaging. In order to achieve a good relative pointing accuracy, we used the bright object MMS 7 (Chini et al. 1997) as a positional standard from which we offset the telescope for each new target position. This enabled us to correlate the MIR sources in a unique way with detections from the VLA, the 2MASS data base, and (sub)mm observations. Several suitable TIMMI 2 standard stars were observed for calibration purposes.

The $1200 \mu \mathrm{m}$ continuum observations were carried out with the 37-channel bolometer array SIMBA at the SEST on La Silla, Chile during the second commissioning period in October 2001. Skydips were performed every three hours in order to correct for the atmospheric opacity. Maps of Uranus were taken for calibration purposes. The final map of about $1^{\circ}$ in declination covers the full region of OMC 1 to 3 and was created from 13 single fast-scanning maps. The residual noise is about $40 \mathrm{mJy} /$ beam (rms). The beam size is $24^{\prime \prime}$. All data were

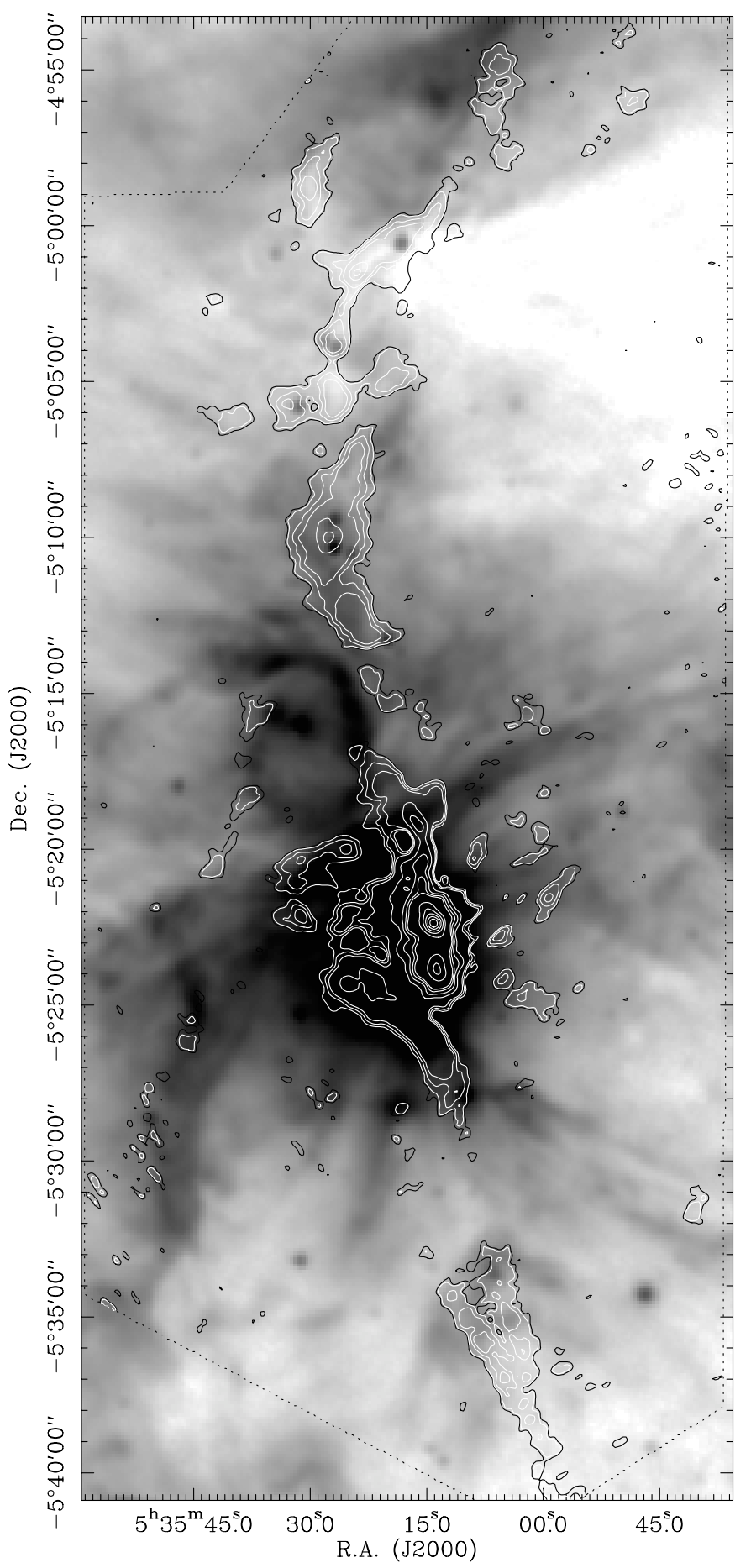

Fig. 1. A SIMBA view of the Orion A region at $1200 \mu \mathrm{m}$ (contours) superimposed on an MSX image at $8.3 \mu \mathrm{m}$ (grey-scale). In the Northern region, dust filaments that are seen in emission with SIMBA appear in absorption for MSX, indicating the presence of cool and dense dust. The central part around the Kleinmann-Low nebula in OMC 1 is dominated by MIR emission suggesting considerably higher dust temperatures.

reduced and analysed with MOPSI ${ }^{1}$ according to the instructions of the SEST manual (2002).

\footnotetext{
${ }^{1}$ MOPSI is a reduction software for infrared, millimetre and radio data, developed and constantly upgraded by R. Zylka, IRAM, Grenoble, France.
} 


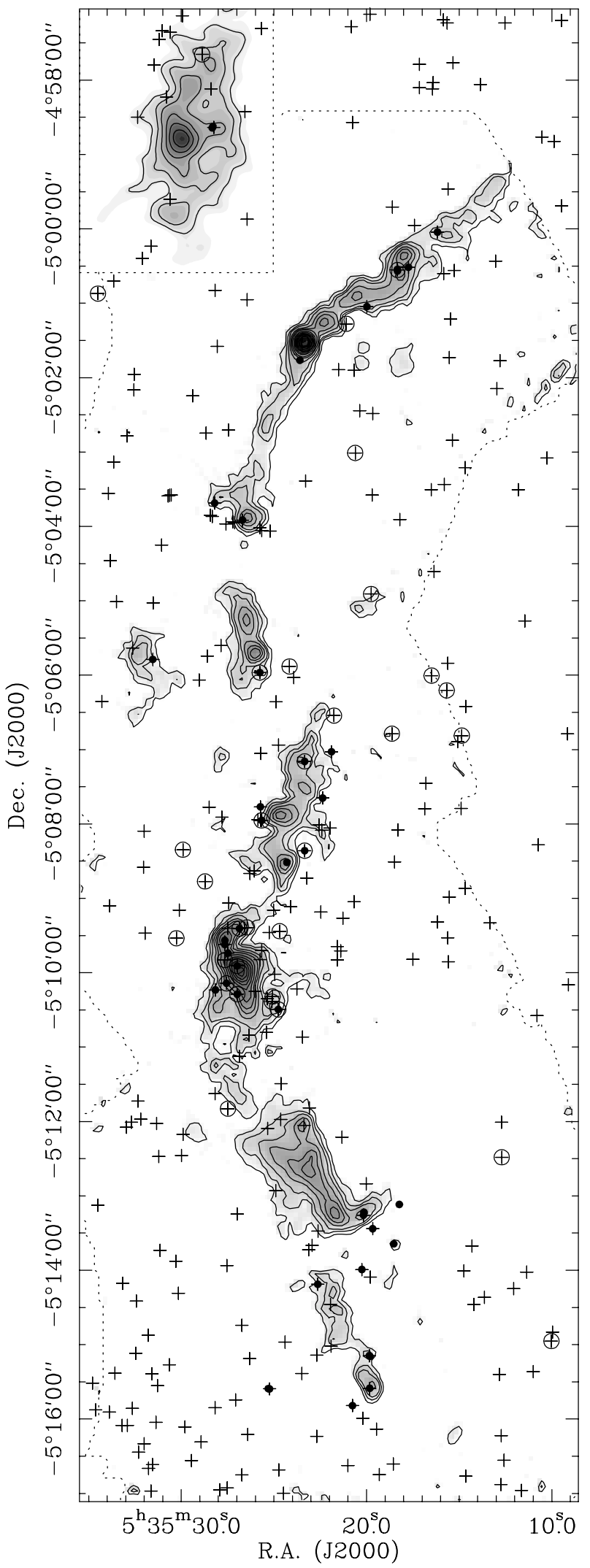

Fig. 2. The OMC $2 / 3$ map at $1.3 \mathrm{~mm}$ by Chini et al. (1997) is shown as a grey-scale enhanced with contours rising linearly from a $3 \sigma \mathrm{rms}$ noise level. Its Southern extension of $3^{\prime}$ was kindly provided by A. Sievers. The upper left insert was taken from the SIMBA map. Our new MIR sources from TIMMI 2 are marked as filled dots while the 2MASS sources show up as crosses. Objects with a colour excess are pointed out with an open circle.
In addition, we show an extension of the spatially higher resolved $1300 \mu \mathrm{m}$ image of Chini et al. (1997) of 3' towards the South. This hitherto unpublished map was obtained at IRAM (Sievers, priv. comm.) and allowed us to look for coinciding detections down to a declination of $-5^{\circ} 16^{\prime} 30^{\prime \prime}$.

The 2MASS data were accessed via the VizieR Online Catalogue Service ${ }^{2}$. Only such objects were selected which were detected in at least two wavebands of $J H K_{\mathrm{s}}$. The cited $K_{\mathrm{s}}$ magnitude was transformed into a $K$ magnitude according to Wainscoat \& Cowie (1992). Furthermore, the conversion from magnitudes to physical flux quantities was achieved following the calibration information of the 2MASS Explanatory Supplement (Sect. IV 5a).

\section{Overview of the region}

Figure 1 gives an overview of the Orion A region including OMC 1-3; it was obtained with SIMBA at $1200 \mu \mathrm{m}$ and extends the original map by Chini et al. (1997) both towards North and South. This map resembles - apart from the lower resolution - very much our previous one, but shows this time fainter extended emission. In addition, there are new complexes with embedded sources in the Northern and the very Southern region; the strongest compact source to the very North-East is labelled "S" according to "SIMBA". The $1200 \mu$ m contours are plotted over an $8.3 \mu \mathrm{m}$ image from MSX (Midcourse Space Experiment). The correspondence at both wavebands is striking. The $1200 \mu \mathrm{m}$ emission filaments in OMC 3 appear in absorption in the MSX map, indicating the presence of dense and cool dust. Moving further to the South, the dust temperature seems to increase as witnessed by the emission at $8.3 \mu \mathrm{m}$.

Figure 2 shows the $1300 \mu \mathrm{m}$ emission of OMC $2 / 3$ at twice the resolution of Fig. 1; it was taken from Chini et al. (1997) and was extended to the South by an additional mosaic of $3^{\prime}$ in declination, kindly provided by A. Sievers (priv. comm.). The positions of the new MIR sources from TIMMI 2 as well as all 2MASS detections within the region are also marked. It becomes clear from this figure that we have to deal with a number of combinations as concerns the coincidence of detections at NIR, MIR and mm wavelengths.

Table 1 gives the positions for all objects detected in our MIR survey. Although there is a number of different names associated with certain sources in the field, we use - for reasons of homogeneity - the running number of the MIR sources as a nomenclature in the present paper. Other designations are given for comparison. Comparing the positions derived for our $10.4 \mu \mathrm{m}$ sources with astrometry from the VLA and 2MASS, we estimate an accuracy of better than $1^{\prime \prime}$. The VLA positions are taken from Reipurth et al. (1999), while the NIR reference frame is based on the 2MASS database. Whenever the the nominal MIR position according to the telescope information agreed within $3^{\prime \prime}$ with a VLA or a 2MASS detection, we assumed that the sources are identical. Since many MIR frames contain more than one object and are often overlapping with adjacent frames, the identifications could be

\footnotetext{
${ }^{2}$ http://vizier.u-strasbg.fr
} 
cross-checked against each other and with the MSX data, giving a consistent picture.

Altogether, there are 45 MIR sources which have 28 counterparts in the $1300 \mu \mathrm{m}$ map by Chini et al. (1997). However, only the 10 strongest mm sources were labelled with an MMS or FIR number by Chini et al. (1997) while 17 weaker mm sources do not yet have any millimetre designation. Those sources are marked with a "+" in Table 1 in order to emphasise the coincidence between a MIR and a $1300 \mu \mathrm{m}$ source.

The NIR identifications in Table 1 refer to scan No. 10 of the 2MASS survey as well as the measurements of Ali \& DePoy (1995, AD), Carpenter et al. (2001, CHS) and Gatley et al. (1974), who introduced the labelling of IRS 1 to 5. Additionally, those IRAS sources are mentioned, whose error ellipses include the positions of our MIR sources.

The photometric data is given in Table 2. It contains the NIR flux densities, mostly taken from the 2MASS database. In rare cases, where 2MASS data were not available, we used the measurements of AD and CHS. The listed MIR flux densities were acquired with our TIMMI 2 observations. For reasons of homogeneity, we re-measured all $1300 \mu \mathrm{m}$ flux densities for the MIR sources. Therefore, the values for those 15 objects which were not obviously outstanding in the $1300 \mu \mathrm{m}$ map by Chini et al. (1997) are new and are marked in bold letters. The same holds for the new North-Eastern source, where we extracted the millimetre flux from the SIMBA map. The previously published values are in accord with our new estimates.

\section{Results}

The detection of an individual source at the different wavebands (see Table 1) gives a first indication that we are dealing with a variety of sources at different evolutionary stages. Likewise, Table 2 corroborates this view, because the relative energy output at certain spectral regions changes tremendously from source to source. In order to get a comprehensive view of the stellar content in the region, we have used two major data sets: 43 sources come from our MIR survey (hereafter MIR sample) whereas 264 sources were obtained from the 2MASS database (NIR sample). Subsequently, both groups were divided according to their location in OMC 2 and 3, respectively.

\subsection{The JHK colour-colour diagram}

Figure 3 shows the NIR colour-colour diagram of all 264 known IR sources in the region; the photometric errors in both axes are typically around $0.2 \mathrm{mag}$, and rise up to $1 \mathrm{mag}$ for the faintest objects. Despite this large error margin, the sources are mainly located in "allowed" regions as discussed below. Unfortunately, not all MIR sources can be associated with NIR counterparts: 5 MIR binary systems have not been resolved by 2MASS and thus have a single NIR entry only. 2 MIR objects were exclusively detected at $K_{\mathrm{s}}$ and 2 MIR objects could not be observed at all. In order to achieve a crude classification of the sources we distinguish four different areas within the $J H K$ diagram.

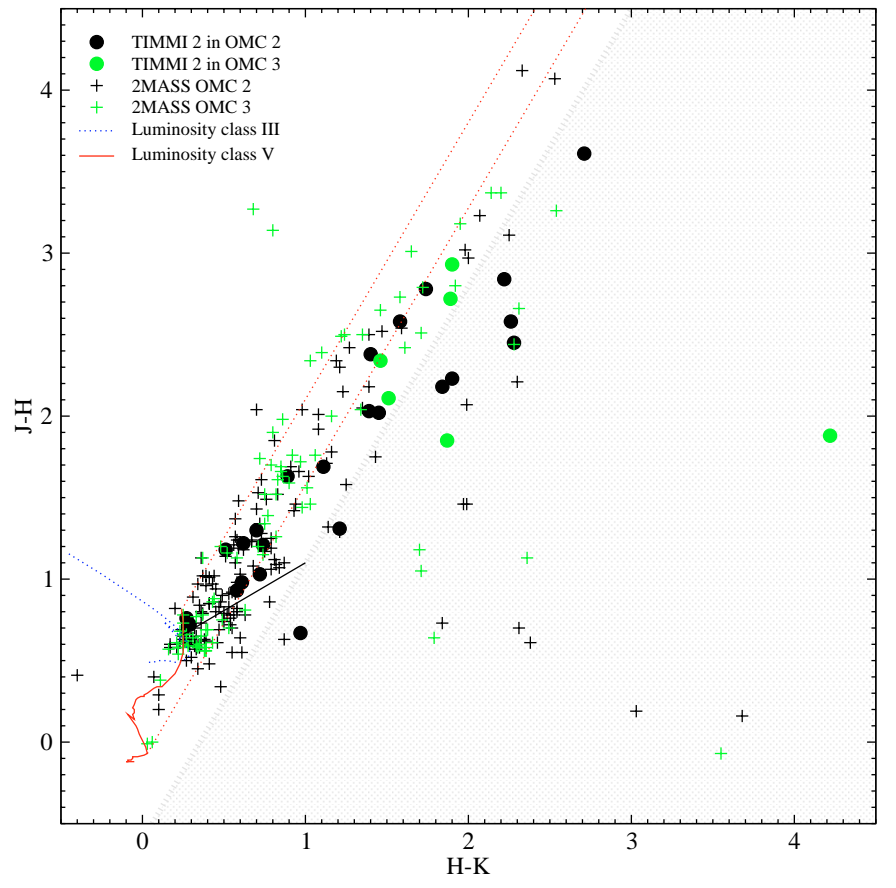

Fig. 3. The NIR colour-colour diagram of 264 sources in the OMC $2 / 3$ region. The symbols follow our division into a MIR $(\bullet)$ and a NIR (+) sample; black symbols refer to sources in OMC 2, grey symbols to those in OMC 3. The unreddened loci of MS stars (solid) and giants (dotted) according to Ducati et al. (2001) are indicated as well as the locus of unreddened T Tauri stars, as derived by Meyer et al. (1997). The straight dotted lines denote the direction of the normal reddening vector, the shaded area depicts the region of NIR colour excesses.

\subsubsection{Giant region}

Within the errors, 4 NIR sources lie in the region of giant stars. 3 of them have photometry errors of $>0.4$ mag which makes a secure classification as giant stars rather uncertain. Since 2 sources have extinction values $15<A_{V}<20 \mathrm{mag}$, we regard them as true background objects not related to the stellar population of the OMC 2/3 region. These 4 objects were excluded from any further analysis.

\subsubsection{Main-sequence region}

There are 172 sources with "normal" colours in the area of reddened main-sequence (MS) stars with $A_{V}$-values of up to 30 mag. Following our crude division, the majority of objects $(161 \equiv 61 \%)$ comes from the NIR sample only, indicating that this population is dominated by evolved objects. On the other hand, only 11 objects (37\%) of the MIR sample have NIR colours that are compatible with MS stars.

\subsubsection{T Tauri region}

Taking - somewhat arbitrarily - the lower reddening vector for MS stars as the dividing line between MS and T Tauri (TT) stars we end up with 57 sources that are located in the T Tauri region. They exhibit $A_{V}$-values of up to $30 \mathrm{mag}$. Here, 48 objects $(18 \%)$ come from the NIR sample alone. 9 objects 
Table 1. Source positions and other designations of the new MIR sources. The braced brackets indicate those double sources which were not resolved by the millimetre and/or the NIR observations. The millimetre detections are marked in the column "MM" according to Chini et al. (1997); those millimetre peaks without a previous designation are marked as "+"; "S(c)" indicates the "c" component of the the new mm detection with SIMBA. Two identifications (FIR 4 and 5) are uncertain and thus marked as "(?)". The sources listed in the column "CSO" are taken from Lis et al. (1998) and the column "VLA" contains detections published by Reipurth et al. (1999). Coinciding NIR and FIR sources are listed in the next five columns. The "2MASS" numbers are from 2MASS scan No. 10; "AD" and "CHS" numbers are taken from Ali \& DePoy (1995) and Carpenter et al. (2001). The "IRS" sources were published in Gatley et al. (1974); IRAS sources, whose positions match within their error ellipses, are also mentioned. The sources MIR 12 and 13 represent the borderline between OMC 2 and 3.

\begin{tabular}{|c|c|c|c|c|c|c|c|c|c|c|}
\hline \multirow[b]{2}{*}{ MIR } & RA & \multirow{2}{*}{$\begin{array}{ll}00) & \text { Dec } \\
& \end{array}$} & \multicolumn{8}{|c|}{ other designations } \\
\hline & $(\mathrm{J} 2000)$ & & MM & $\mathrm{CSO}$ & VLA & 2MASS & $\mathrm{AD}$ & CHS & IRS & IRAS \\
\hline 1 & $5^{\mathrm{h}} 35^{\mathrm{m}} 28^{\mathrm{s}} .29$ & $-4^{\circ} 58^{\prime} 37^{\prime \prime} .5$ & & & & & & & & \\
\hline 2 & 28.33 & $58^{\prime} 38^{\prime \prime} .5$ & S(c) & & & 2083 & & 10398 & & \\
\hline 3 & 16.17 & $-5^{\circ} 00^{\prime} 02^{\prime \prime} 6$ & + & 3 & & 2068 & & 8787 & & \\
\hline 4 & 17.74 & $00^{\prime} 31^{\prime \prime} .9$ & & & & 2063 & & & & \\
\hline 5 & 18.34 & $00^{\prime} 32^{\prime \prime} .8$ & & & 1 & & & & & \\
\hline 6 & 18.33 & $00^{\prime} 34^{\prime \prime} 0$ & MMS 2 & 6 & & 2062 & & & & \\
\hline 7 & 19.98 & $01^{\prime} 02^{\prime \prime} .9$ & MMS 4 & 8 & & 2053 & & & & \\
\hline 8 & 23.6 & $01^{\prime} 46^{\prime \prime}$ & & & & & & & & \\
\hline 9 & 28.31 & $03^{\prime} 40{ }^{\prime} 8$ & + & 11 & & 2014 & & & & \\
\hline 10 & 26.69 & $03^{\prime} 54^{\prime \prime} .9$ & MMS 7 & 12 & 4 & 2006 & 2117 & & & 05329-0505 \\
\hline 11 & 31.54 & $05^{\prime} 47^{\prime \prime} .3$ & + & & & 1979 & 2468 & 10792 & & \\
\hline 12 & 25.76 & $05^{\prime} 57^{\prime \prime} .9$ & + & & & 1975 & 2079 & & & \\
\hline 13 & $21^{\mathrm{s}} .89$ & $07^{\prime} 01^{\prime \prime} .8$ & & & & 1957 & & 9617 & & $05329-0508$ \\
\hline 14 & 23.34 & $07^{\prime} 09 ! 8$ & FIR 1c & 16 & & 1954 & 2066 & & & \\
\hline 15 & 22.37 & $07^{\prime} 399^{\prime} 1$ & & & & 1948 & 2064 & & & \\
\hline 16 & 25.73 & $07^{\prime} 46^{\prime \prime} .2$ & & & & 1946 & & 10104 & & \\
\hline 17 & 25.58 & $07^{\prime} 57^{\prime \prime} .4$ & & & 9 & 1936 & 2062 & & & \\
\hline 18 & $23^{\S} .34$ & $08^{\prime} 21^{\prime \prime} 6$ & & & & 1925 & 1928 & & & \\
\hline 19 & 24.30 & $08^{\prime} 31^{\prime \prime} 0$ & FIR 2 & 20 & & & & & & \\
\hline 20 & 26.86 & $09^{\prime} 24^{\prime \prime} 4$ & + & & & 1896 & & 10242 & 2 & \\
\hline 21 & 27.65 & $09^{\prime} 34^{\prime \prime} 0$ & & & 11 & & 2446 & & $4-\mathrm{N}$ & \\
\hline 22 & 27.65 & $09^{\prime} 37^{\prime \prime} .2$ & FIR 3 & 22 & & 1886 & & 10318 & $4-S$ & \\
\hline 23 & 27.48 & $09^{\prime} 44^{\prime \prime} .2$ & & & & 1882 & 2445 & & & \\
\hline 24 & 26.99 & $09^{\prime} 54^{\prime \prime} .5$ & FIR 4(?) & 23 & 12 & 1876 & 1867 & & & \\
\hline 25 & 27.56 & $10^{\prime} 08^{\prime \prime} .5$ & + & & & 1868 & 1863 & & & \\
\hline 26 & 28.16 & $10^{\prime} 13^{\prime \prime} .9$ & + & & & 1864 & 2996 & 10383 & & \\
\hline 27 & 26.98 & $10^{\prime} 17^{\prime \prime} .3$ & FIR 5(?) & 24 & & 1862 & 2444 & & 3 & 05329-0512 \\
\hline 28 & 24.76 & $10^{\prime} 29.6$ & + & & 13 & 1854 & 2443 & & 1 & \\
\hline 29 & 23.37 & $12^{\prime} 03^{\prime \prime} 0$ & FIR 6b & 25 & & 1822 & 1691 & & & \\
\hline 30 & 18.23 & $13^{\prime} 06^{\prime \prime} 9$ & + & & & & 2440 & 9094 & & \\
\hline 31 & 20 s.14 & $13^{\prime} 13^{\prime \prime} .2$ & & & & & 2405 & & & \\
\hline 32 & $20 \varsigma 16$ & $13^{\prime} 15^{\prime \prime} 6$ & FIR $6 d$ & 30 & & 1796 & 2439 & 9373 & & \\
\hline 33 & 19.67 & $13^{\prime} 26^{\prime \prime} 5$ & & & & 1793 & 1679 & 9305 & & \\
\hline 34 & 18.53 & $13^{\prime} 38^{\prime \prime} .7$ & & & & & 2404 & 9147 & & \\
\hline 35 & $20 \varsigma 25$ & $13^{\prime} 59 ! 4$ & & & & 1775 & 2403 & & & \\
\hline 36 & $22^{s} .63$ & $14^{\prime} 11^{\prime \prime} .3$ & + & & & 1770 & 1667 & & & \\
\hline 37 & $21^{\mathrm{s}} .94$ & $14^{\prime} 27^{\prime \prime} 6$ & + & 31 & & 1762 & & & & \\
\hline 38 & $21^{\mathrm{s}} .92$ & $15^{\prime} 01^{\prime \prime} .1$ & + & 32 & & 1748 & 1654 & 9624 & & \\
\hline 39 & 19.85 & $15^{\prime} 08^{\prime \prime} .9$ & & & & & & & & \\
\hline 40 & 19.81 & $15^{\prime} 09^{\prime} .5$ & + & & & 1743 & 2402 & 9335 & & \\
\hline 41 & 23.49 & $15^{\prime} 23^{\prime \prime} .3$ & + & & & 1736 & 1646 & 9829 & & \\
\hline 42 & 19.83 & $15^{\prime} 35^{\prime \prime} \cdot 2$ & + & 33 & & 1731 & 1644 & 9333 & & \\
\hline 43 & 25.30 & $15^{\prime} 35^{\prime \prime} .5$ & + & & & & & & & \\
\hline 44 & $25^{5} .24$ & $15^{\prime} 35^{\prime \prime} .7$ & + & & & 1730 & 2401 & 10037 & & \\
\hline 45 & $20 \$ .76$ & $15^{\prime} 49^{\prime} .2$ & & & & 1722 & 2994 & & & \\
\hline
\end{tabular}


Table 2. Photometric data and spectral properties. The MIR $1 \sigma$ noise level of one image from our TIMMI 2 measurements is typically $25 \mathrm{mJy} / \square^{\prime \prime}$ in the $N$-band and about $370 \mathrm{mJy} / \square^{\prime \prime}$ in the $Q$-band. The $350 \mu \mathrm{m}$ data come from Lis et al. (1998), the $1300 \mu \mathrm{m}$ flux densities were determined from the extended map of Chini et al. (1997), and have a $1 \sigma$ noise level of $15 \mathrm{mJy} /$ beam. Newly determined millimetre fluxes are marked in bold letters. VLA data at $3.6 \mathrm{~cm}$ are taken from Reipurth et al. (1999).

\begin{tabular}{|c|c|c|c|c|c|c|c|c|c|c|c|}
\hline \multirow[b]{2}{*}{ MIR } & \multicolumn{8}{|c|}{ 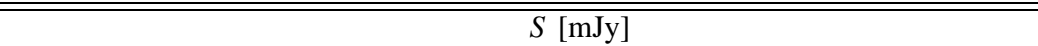 } & \multirow[b]{2}{*}{$3.6 \mathrm{~cm}$} & \multirow[b]{2}{*}{$\alpha_{\mathrm{KN}}$} & \multirow[b]{2}{*}{$L_{\mathrm{bol}} / L_{\mathrm{smm}}$} \\
\hline & $J$ & $H$ & $K_{\mathrm{s}}$ & $10.4 \mu \mathrm{m}$ & $11.9 \mu \mathrm{m}$ & $17.8 \mu \mathrm{m}$ & $350 \mu \mathrm{m}$ & $1300 \mu \mathrm{m}$ & & & \\
\hline $\begin{array}{l}1 \\
2\end{array}$ & $<0.2$ & 2.3 & 18.5 & $\begin{array}{l}30 \\
30\end{array}$ & & & & 290 & & -0.6 & \\
\hline 3 & 0.5 & 5.0 & 18.5 & 180 & 240 & 1270 & 17000 & 179 & & 0.3 & $60-180$ \\
\hline 4 & 1.1 & 6.2 & 15.3 & 25 & 40 & & & & & -0.8 & \\
\hline 5 & 02 & 08 & 44 & 745 & 655 & 1600 & 25000 & 240 & 0.25 & 11 & $370-1350$ \\
\hline 6 & 0.2 & 0.8 & 24.4 & 355 & 200 & 915 & 25000 & 249 & & 1.1 & $5 / 0-1530$ \\
\hline 7 & & & 1.1 & 20 & & & 27000 & 354 & & 0.9 & $10-600$ \\
\hline 8 & & & & 230 & 300 & & & & & & \\
\hline 9 & $<0.8$ & 0.5 & 10.3 & 475 & 445 & 1430 & 15000 & 164 & & 1.2 & $100-755$ \\
\hline 10 & 2.3 & 19.4 & 71.6 & 990 & 1495 & 8830 & 19000 & 360 & 0.59 & 0.7 & 115 \\
\hline 11 & 28.2 & 134.8 & 347.4 & 860 & 2090 & 2260 & & 228 & & -0.1 & $90-260$ \\
\hline 12 & 0.6 & 2.1 & 6.5 & 85 & & & & 146 & & 0.6 & \\
\hline 13 & 64.9 & 109.3 & 124.2 & 245 & & & & & & -0.6 & \\
\hline 14 & 0.3 & 1.7 & 8.6 & 30 & & $<2665$ & 18000 & 180 & 2.84 & -0.3 & $80-995$ \\
\hline 15 & 1.7 & 14.6 & 46.6 & 75 & & $<2665$ & & & & -0.9 & \\
\hline 16 & 2.2 & 9.6 & 23.5 & 20 & & $<2450$ & & & & -1.3 & \\
\hline 17 & 29.8 & 37.6 & 58.4 & 140 & & 790 & & & 0.24 & -0.5 & \\
\hline 18 & 0.4 & 2.2 & 8.0 & 20 & & & & & & 0.2 & \\
\hline 19 & & & & 11 & & & 17000 & 340 & & & $10-600$ \\
\hline 20 & 1.3 & 6.8 & 23.8 & 1510 & 2275 & 3430 & & 351 & & 1.5 & $30-310$ \\
\hline 21 & & & & 315 & 364 & 8665 & & & & 0.0 & $80-645$ \\
\hline 22 & 9.3 & 68.4 & 189.2 & 1130 & 1130 & 1980 & 36000 & $6 / 6$ & & 0.0 & $80-645$ \\
\hline 23 & 30.0 & 67.8 & 83.5 & 140 & 75 & $<2830$ & & & & -0.7 & \\
\hline 24 & 1.3 & 3.0 & 5.9 & 45 & & $<2830$ & 67000 & 1252 & 0.64 & 0.2 & $15-340$ \\
\hline 25 & $<0.6$ & 1.1 & 5.2 & 26 & & & & 216 & & 0.0 & \\
\hline 26 & 52.0 & 91.8 & 115.2 & 332 & & & & 423 & & -0.3 & \\
\hline 27 & 4.9 & 46.2 & 230.0 & 8510 & & & 34000 & 452 & & 1.1 & 160 \\
\hline 28 & 0.6 & 11.2 & 88.7 & 2995 & & 18010 & & 186 & 1.04 & 1.1 & 1075 \\
\hline 29 & $<0.1$ & 0.4 & 5.4 & $<50$ & & & 15000 & 300 & & $<0.5$ & $15-705$ \\
\hline 30 & 40 & 80 & 90 & 60 & & & & 186 & & -1.4 & \\
\hline 31 & & & 170 & 430 & 365 & & & 314 & & -0.4 & $165-2760$ \\
\hline 32 & 176.9 & 781.7 & 1635 & 4060 & 4845 & & 12000 & 314 & & -0.6 & $165-2760$ \\
\hline 33 & 4.0 & 24.3 & 56.8 & 75 & 685 & & & & & -1.0 & \\
\hline 34 & 30 & 80 & 110 & 550 & 92 & & & & & 0.0 & \\
\hline 35 & 17.8 & 54.4 & 79.4 & 60 & & & & & & -1.3 & \\
\hline 36 & 32.4 & 52.2 & 57.2 & 39 & & & & 184 & & -1.4 & \\
\hline 37 & 31.2 & 43.0 & 35.8 & $<65$ & & & 15000 & 189 & & $<-0.6$ & $45-1845$ \\
\hline 38 & 12.1 & 24.4 & 25.2 & $<55$ & & & 15000 & 161 & & $<-0.5$ & $45-1890$ \\
\hline 39 & 9.7 & 31.5 & 56.1 & 255 & & & & 140 & & 0.2 & $765-2170$ \\
\hline 40 & & & & $\begin{array}{l}150 \\
<65\end{array}$ & & & & 42 & & $<0.9$ & \\
\hline $\begin{array}{l}41 \\
42\end{array}$ & $\begin{array}{r}3.2 \\
<0.1\end{array}$ & $\begin{array}{l}4.2 \\
0.7\end{array}$ & $\begin{array}{l}3.4 \\
8.9\end{array}$ & $\begin{array}{r}<65 \\
80\end{array}$ & & & 20000 & $\begin{array}{r}42 \\
288\end{array}$ & & $\begin{array}{r}<0.9 \\
0.2\end{array}$ & $30-1445$ \\
\hline 43 & 24.9 & 51.6 & 65.6 & 44 & & & & 48 & & -0.7 & \\
\hline 44 & 24.9 & 31.0 & & 74 & & & & $\mathbf{4 0}$ & & -0.1 & \\
\hline 45 & 174.5 & 368.0 & 418.5 & 30 & & & & & & -2.8 & \\
\hline
\end{tabular}

of the MIR selected sample (30\%) are found in that region. This suggests that the MIR group contains a larger fraction of T Tauri stars.

\subsubsection{Excess region}

The shaded area in Fig. 3 denotes the regime of IR-excess stars. Its left boundary to the T Tauri regime is determined by the truncation of the T Tauri branch at $H-K=1$, as shown by Meyer et al. (1997). Ten sources were detected at MIR wavelengths $(33 \%)$, whereas $32(12 \%)$ could be found in the NIR. This corroborates the trend that the fraction of YSOs increases in the MIR sample compared to the NIR sample when going to earlier evolutionary stages.

Altogether there are 32 excess objects of which 22 are located in OMC 2 and 10 in OMC 3 . There is no clear division of 


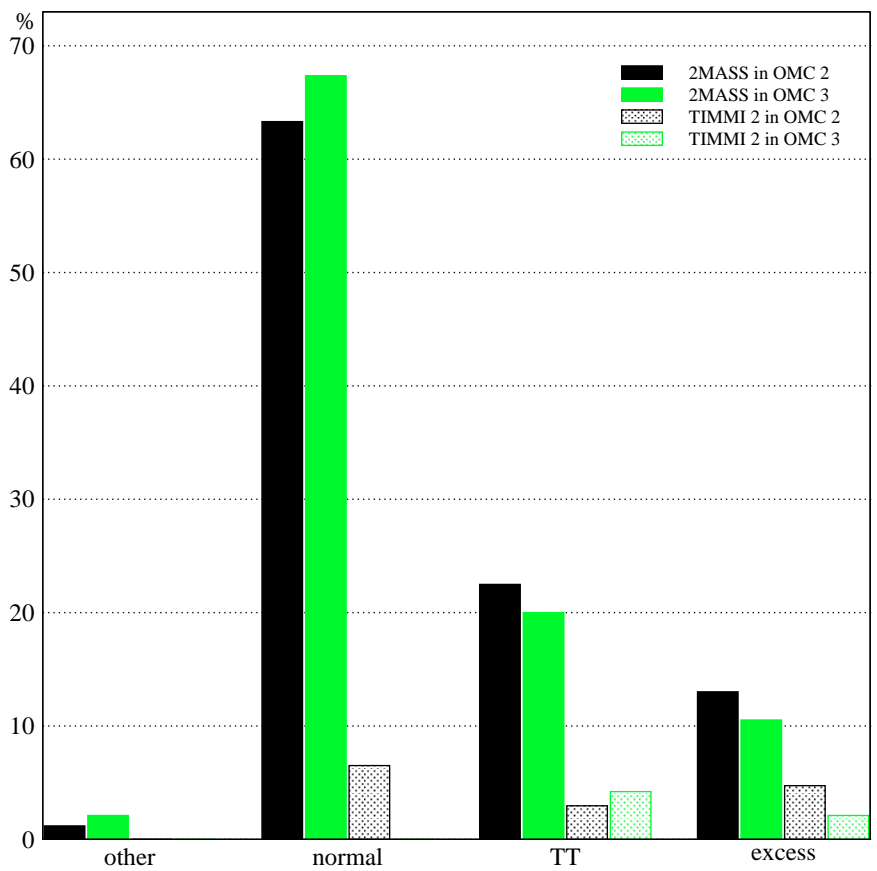

Fig. 4. Statistics of all IR sources as they populate the different areas of the colour-colour diagram. The fractions of sources relative to the total number of IR sources separated in OMC 2 and OMC 3 are given.

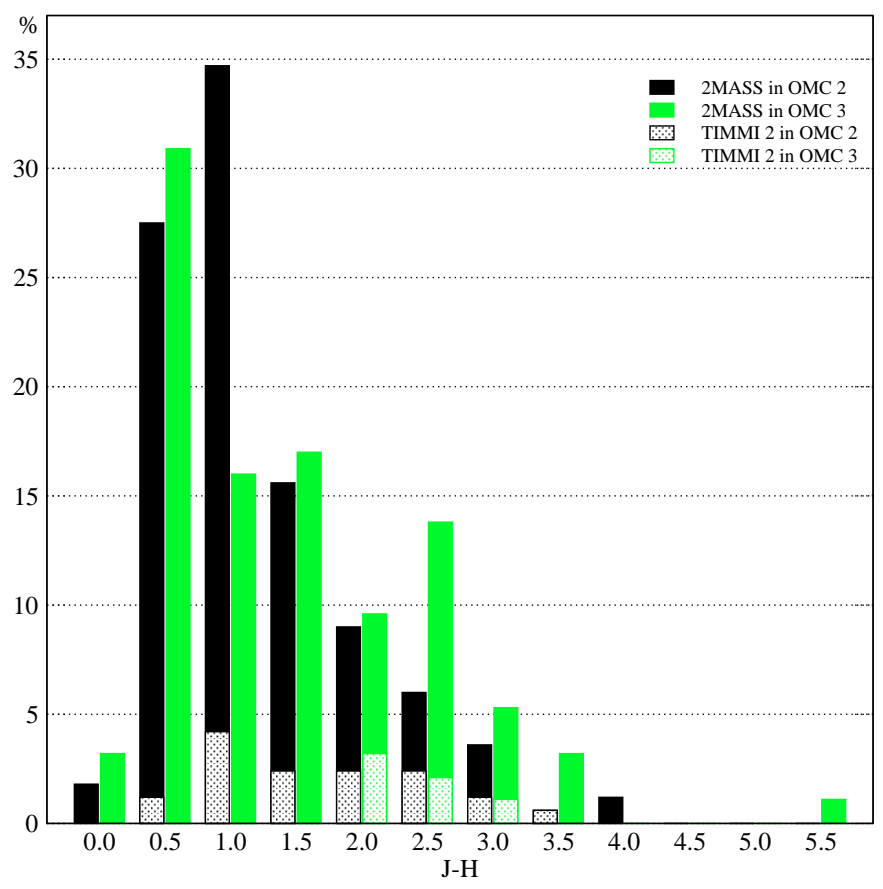

Fig. 5. Relative distribution of $J-H$ colours normalised to the number of 2MASS sources in OMC 2 and 3, respectively.

both groups in terms of extinction and/or strength of excess, although sources in OMC 3 seem to be slightly redder. For example, the source with the highest extinction is the MIR $1 / 2$ binary with $J-H>5$, but it has been excluded from the colour-colour diagram due to its large error. The largest excess of $H-K>4$ also occurs with an OMC 3 object.

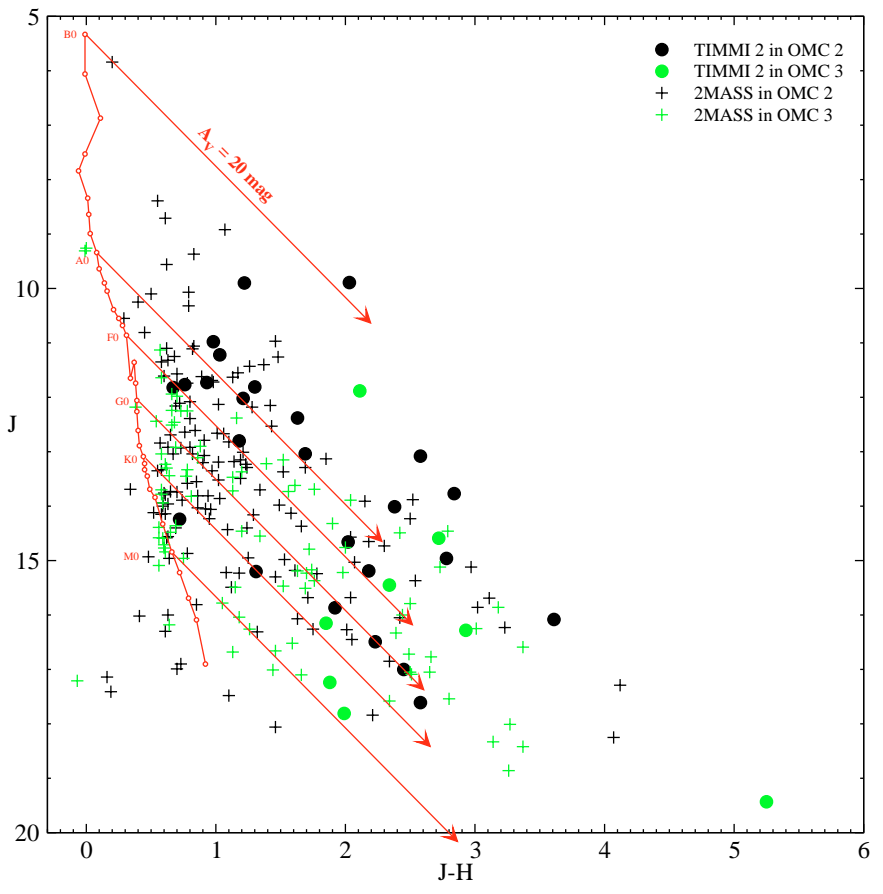

Fig. 6. The NIR colour-magnitude diagram of 260 sources in the OMC 2/3 region, excluding the 4 sources lying in the area of giants. The symbols are as in Fig. 3. The MS has been adjusted for a uniform foreground reddening of $A_{V}=2 \mathrm{mag}$ and a distance of $450 \mathrm{pc}$. Reddening vectors of a length corresponding to $A_{V}=20$ mag were attached to the loci of unreddened types B0 to M0.

Table 3. Statistics of sources in the four colour regions.

\begin{tabular}{ccccc}
\hline \hline & \multicolumn{4}{c}{ Colour region } \\
Sample & other & normal & T Tau & excess \\
\hline 2MASS/OMC 2 & 2 & 107 & 38 & 22 \\
2MASS/OMC 3 & 2 & 64 & 19 & 10 \\
TIMMI 2/OMC 2 & 0 & 11 & 5 & 8 \\
TIMMI 2/OMC 3 & 0 & 0 & 4 & 2 \\
\hline
\end{tabular}

\subsection{The $J$ vs. $J-H$ colour-magnitude diagram}

Figure 6 shows the $J$ vs. $J-H$ colour-magnitude diagram of 260 of the 264 sources excluding the 4 objects populating the area of giant stars in Fig. 3; the symbols are the same as in Fig. 3. The vertical curve represents the location of MS stars from spectral types B0 to M4 (Schmidt-Kaler 1982; Ducati et al. 2001), corrected for a foreground extinction of $A_{V}=2 \mathrm{mag}$ and a distance of $450 \mathrm{pc}$; the arrows correspond to reddening vectors of $A_{V}=20 \mathrm{mag}$. While a few objects experience an extinction of up to $A_{V}=45 \mathrm{mag}$, most of them have $5<A_{V}<25$ without any obvious distinction between OMC 2 and 3 , as visible from Fig. 5 . Within the errors, all $J-H$ colours except $J-H=1.0$ and 2.5 seem to be equally populated between OMC 2 and 3 with a slight trend to a stronger reddening in OMC 3. However, we do not think that these outliers justify the interpretation of a strong indication for OMC 3 sources to have redder colours than OMC 2 sources.

As witnessed from the colour-colour diagram in Fig. 3, a number of sources exhibits strong IR-excesses. Assuming that this effect mainly dominates the $H-K$ colours, Fig. 6 should 


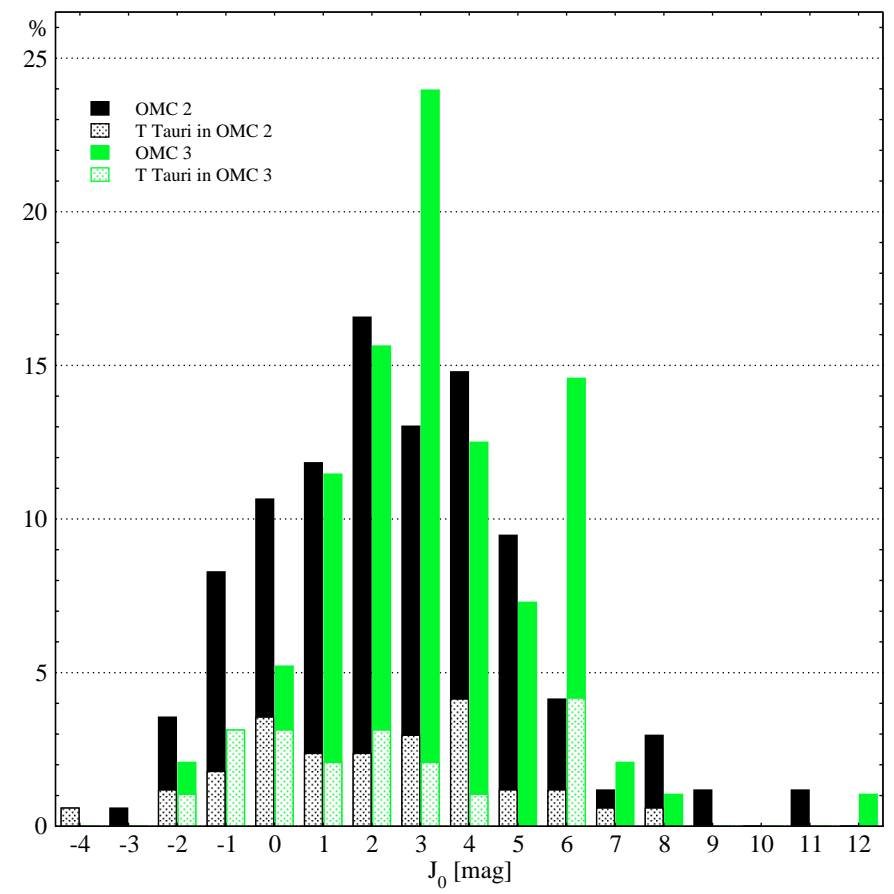

Fig. 7. Relative distribution of $J$ luminosities normalised to the number of 2MASS sources in OMC 2 and 3, respectively.

minimise the influence of the excesses and thus yield reliable results.

All sources show a wide spread in magnitude and colour. The truncation at $J \sim 18 \mathrm{mag}$ is due to the detection limit of the 2MASS observations. Likewise, the photometric errors increase with fainter magnitudes. It is clear that the assignation of spectral types and intrinsic extinction from a colourmagnitude diagram assumes that there are neither foreground nor pre-main sequence stars; both would simulate earlier spectral types. Background stars - in contrast - appear as later types. Unfortunately, neither uncertainty can be removed from the present data set.

In order to minimise these uncertainties, we do not create a distribution of spectral types, but construct a $J$ luminosity function of all stars from Fig. 6 by de-reddening the objects and correct for a distance of $450 \mathrm{pc}$. This analysis should be fairly independent of any colour excess, however it assumes that the stars are on or close to the main sequence. In case of premain sequence objects, the luminosity will be overestimated. On the other hand, the commonly used $K$ luminosity function always simulates higher luminosities, because it may be influenced by both an excess caused by circumstellar emission and by pre-main sequence evolution; both effects may increase the observed $K$ luminosity considerably. Figure 7 displays the fraction of objects in a certain $J$ luminosity interval relative to the total number of 2MASS sources in OMC 2 and 3, respectively. The populations in both regions are distributed across a luminosity range of $-4<M_{J}<12$ with a maximum between $2<M_{J}<3$. The decrease of sources beyond this maximum is certainly due to sensitivity. Nevertheless, there seems to be a systematic shift between the two regions in the sense that OMC 3 contains sources of lower luminosity. Neglecting the pre-main sequence objects from the T Tauri region (dotted), the difference becomes even more pronounced: the fraction of high luminosity, early type stars is significantly higher in OMC 2. Taking into account that OMC 1 - the most Southern region of the Orion A complex - has a rich abundance of massive stars, it seems that there is a decrease of stellar masses along the filament when going from South to North.

\section{Discussion}

In the following we discuss some particular sources of interest either because of their morphology, multiplicity or their detection at other wavelengths as well as the global properties of the association.

\subsection{Individual sources}

\subsubsection{OMC 3 SIMBA}

The new, North-Eastern complex (OMC 3 SIMBA) has also been observed by Lis et al. (1998) and Johnstone \& Bally (1999). Molecular line measurements (source 2 of Tatematsu et al. 1993) allowed to determine its gas mass to $140 M_{\odot}$. In order to determine the dust temperature, we compare this CO result with the total flux density at $1200 \mu \mathrm{m}$ of $3.4 \mathrm{Jy}$ and adopt a mass absorption coefficient $\kappa_{v}$ of $0.03 \mathrm{~cm}^{2}$ per gram of dust. The resulting dust temperature is then $11 \mathrm{~K}$ suggesting that OMC 3 SIMBA is a fairly cold region; this picture is corroborated by the fact that the complex shows up in absorption in the MSX images (see Fig. 1).

As marked in Fig. 8, OMC 3 SIMBA contains at least five condensations, labelled a-e (see also Table 4). The $350 \mu \mathrm{m}$ map of Lis et al. (1998) only shows the brightest component while the more sensitive SCUBA maps of Johnstone \& Bally (1999) resolve the structure, too. Unfortunately, the authors did not elaborate on single sources in their paper, but rather concentrated on the general structure of the filament. Assuming that the total $350 \mu \mathrm{m}$ flux density of $15 \mathrm{Jy}$ originates from component "a", and taking the peak intensity at $1200 \mu \mathrm{m}$ of $570 \mathrm{mJy} / \mathrm{beam}$, we obtain a sub-millimetre luminosity of $0.3 L_{\odot}$. The spectral slope appears to be flat with $\beta=0.7$. Using the above derived dust temperature of $11 \mathrm{~K}$ we derive a gas mass of $24 M_{\odot}$. However, it is likely that much of the underlying extended emission contributes to the peak flux which overestimates the flux density of the compact component "a". Fitting e.g. a 2-dimensional Gaussian with an axis ratio of $33^{\prime \prime} \times 24$ " to component "a" we derive a peak intensity of $375 \mathrm{mJy} / \mathrm{beam}$ and a total flux of $540 \mathrm{mJy}$.

As far as we can judge from the astrometric accuracy, there is a mid-infrared binary source (MIR 1/2) that coincides with component "c". The two objects are of equal strength at $10.4 \mu \mathrm{m}$; their angular separation is 1. .' 2 , and the position angle is $30^{\circ}$, measured clockwise from North. Using the combined NIR colours from 2MASS, this binary system is compatible with spectral type B. 


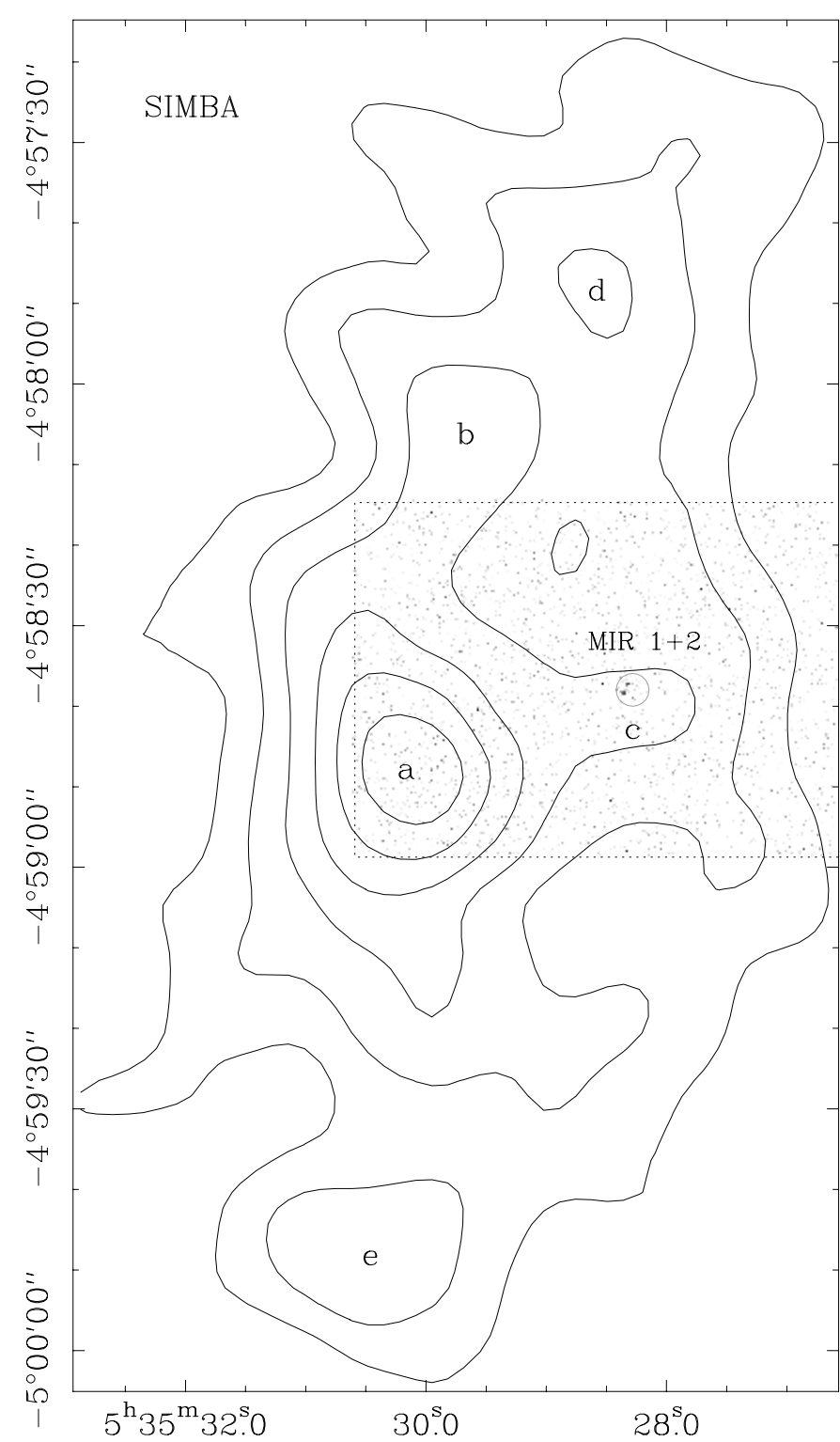

Fig. 8. Superposition of the TIMMI 2 image (rectangular greyscale region) and the SIMBA image (contours) of the region around OMC 3 SIMBA. The TIMMI 2 image shows a binary source (MIR 1/2) that coincides with an extension from the main millimetre source, here labelled as "c".

\subsubsection{OMC 3 MMS2}

The millimetre source OMC 3 MMS 2 contains another MIR binary system (MIR 5/6) as revealed by our TIMMI 2 imaging (see Fig. 9). The projected spatial separation of the components is $1^{\prime \prime} 3$ or $570 \mathrm{AU}$ with a position angle of $45^{\circ}$. Both components of this binary system are surrounded by extended emission at $10.4 \mu \mathrm{m}$. Subtracting the stellar point sources, the residual emission suggests the presence of two separate circumstellar discs. Tsujimoto et al. (2002) resolved this binary with $J H K L$ photometry. They find a colour excess for both sources corroborating the presence of circumstellar material. We will propose high-resolution millimetre interferometry follow-up observations in order to assess the actual distribution of the surrounding material.
Table 4. Positions of millimetre condensations of OMC 3 SIMBA. The flux values are the peak intensities of the source positions in the map. The $1 \sigma$ error is estimated to $30 \mathrm{mJy} / \mathrm{beam}$.

\begin{tabular}{|c|c|c|c|}
\hline & RA (J2000) & 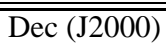 & (S[mJy/beam] \\
\hline $\mathrm{a}$ & $5^{\mathrm{h}} 35^{\mathrm{m}} 30^{\mathrm{s}} \cdot 2$ & $-4^{\circ} 58^{\prime} 48^{\prime \prime}$ & 570 \\
\hline$b$ & 29.7 & $58^{\prime} 06^{\prime \prime}$ & 330 \\
\hline $\mathrm{c}$ & 28.2 & $58^{\prime} 40^{\prime \prime}$ & 290 \\
\hline $\mathrm{d}$ & 28.6 & $58^{\prime} 48^{\prime \prime}$ & 290 \\
\hline $\mathrm{e}$ & 30.6 & $59^{\prime} 49^{\prime \prime}$ & 270 \\
\hline
\end{tabular}

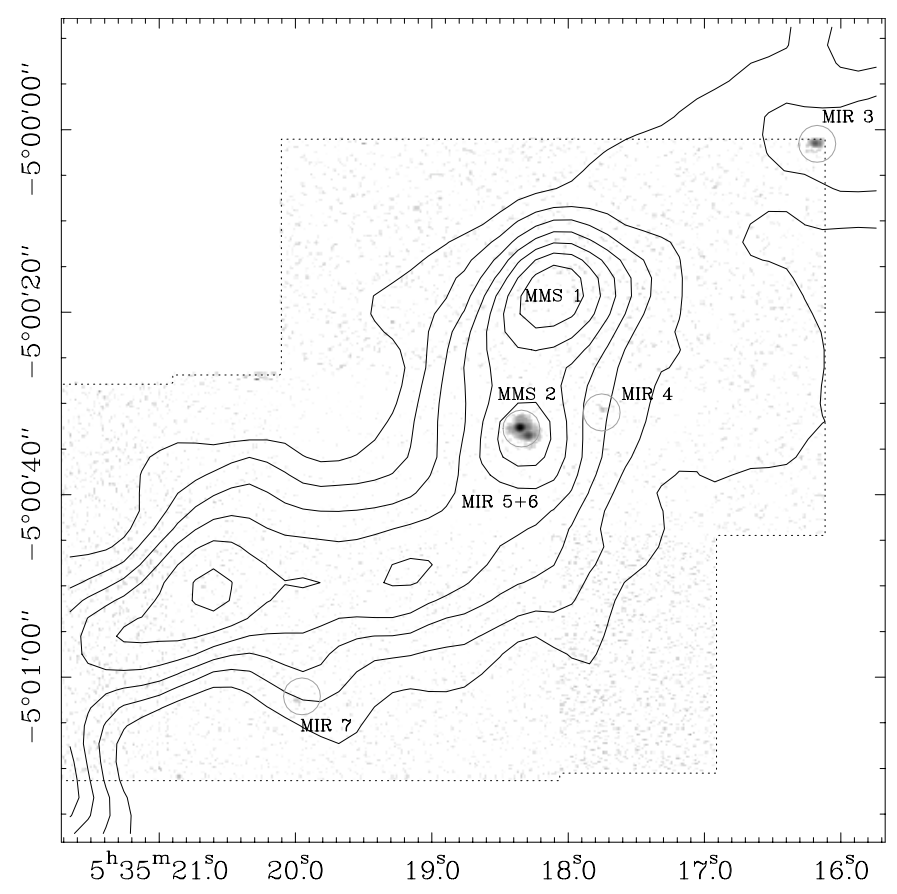

Fig. 9. Superposition of the MIR and $\mathrm{mm}$ data of the region around OMC 3 MMS 2 (MIR 5/6). The grey-scale picture is the TIMMI 2 image showing three single and one binary source. The contours are taken from the millimetre map of Chini et al. (1997).

By applying Gaussians fits to the TIMMI 2 sources, we obtain similar ellipticities for both which would correspond to an inclination angle of $33^{\circ} \pm 2^{\circ}$ relative to the plane of the sky. The combined NIR colours classify this system as $K$ - the only late type binary system found in our sample - with the largest excess of $H-K \sim 4$. Analysing the two single YSOs individually, Tsujimoto et al. (2002) find similar excess values for both. The colours from this study point to spectral types of A or B. From the spectral index $\alpha$, we conclude that both components are of Class I.

MMS 2 coincides with the X-ray source 8 (Tsuboi et al. 2001) while MIR 5 is obviously identical to the X-ray source $8 \mathrm{c}$. The X-ray spectrum also shows evidence for two different sources, one of which has a soft the other one a hard spectrum. Tsuboi et al. (2001) interpret sources with soft X-ray emission as T Tauri stars whereas hard X-ray emission is characteristic for outflows and/or jets. Williams et al. (2003) found a very long $\mathrm{CO}$ outflow associated with MMS 2 .

Another MIR source (MIR 7) cannot be related to any of the millimetre clumps in that region but it coincides with the 


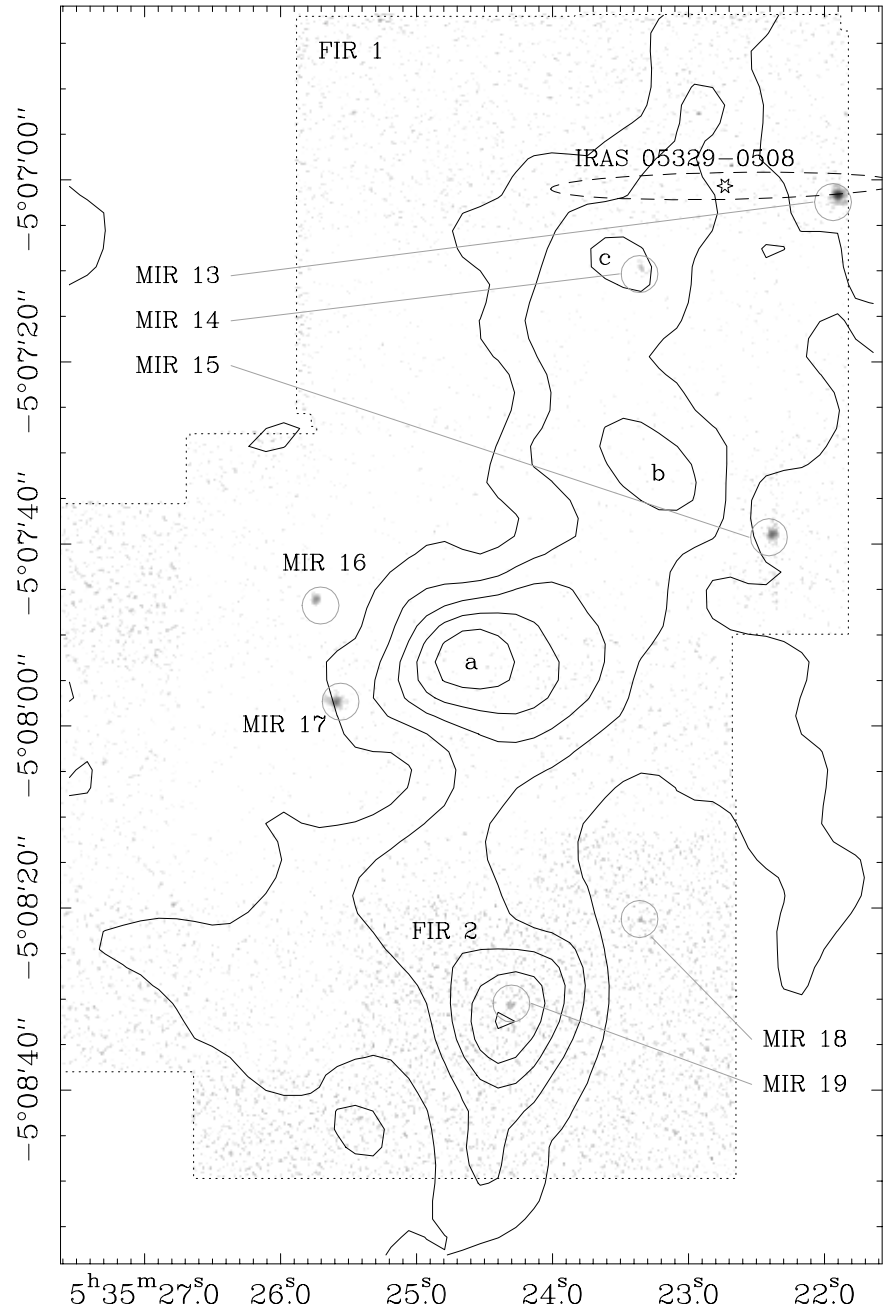

Fig. 10. Superposition of the MIR and $\mathrm{mm}$ data of the region around OMC 2 FIR 1 (MIR 13-19). The grey-scale picture is the TIMMI 2 image showing 7 sources. The contours are taken from the millimetre map of Chini et al. (1997).

X-ray source 12 of Tsuboi et al. (2001). Its JHK colours classify the star as T Tauri which is compatible with its location far above the MS in the $J$ vs. $J-H$ colour-magnitude diagram. The spectral index $\alpha$ is compatible with Class I.

\subsubsection{OMC 2 FIR 1-2}

The FIR 1-2 complex harbours seven MIR sources, two of which (MIR 14 and 19) are embedded in distinct $1200 \mu \mathrm{m}$ condensations. MIR 14 has the strongest excess in OMC 2 with a $\Delta K$ of $\sim 1 \mathrm{mag}$ and an $M_{J}$ of 3.2 ; from its spectral index it is classified as Class II. MIR 19 was not detected by 2 MASS.

\subsubsection{OMC 2 FIR 3-5}

Within the FIR 3-5 complex we could detect nine MIR sources (MIR 20-28) of which five are associated more or less with millimetre emission (see Fig. 11). MIR 20 lies within an unresolved North-Western extension of FIR 3; its $J H K$ colours

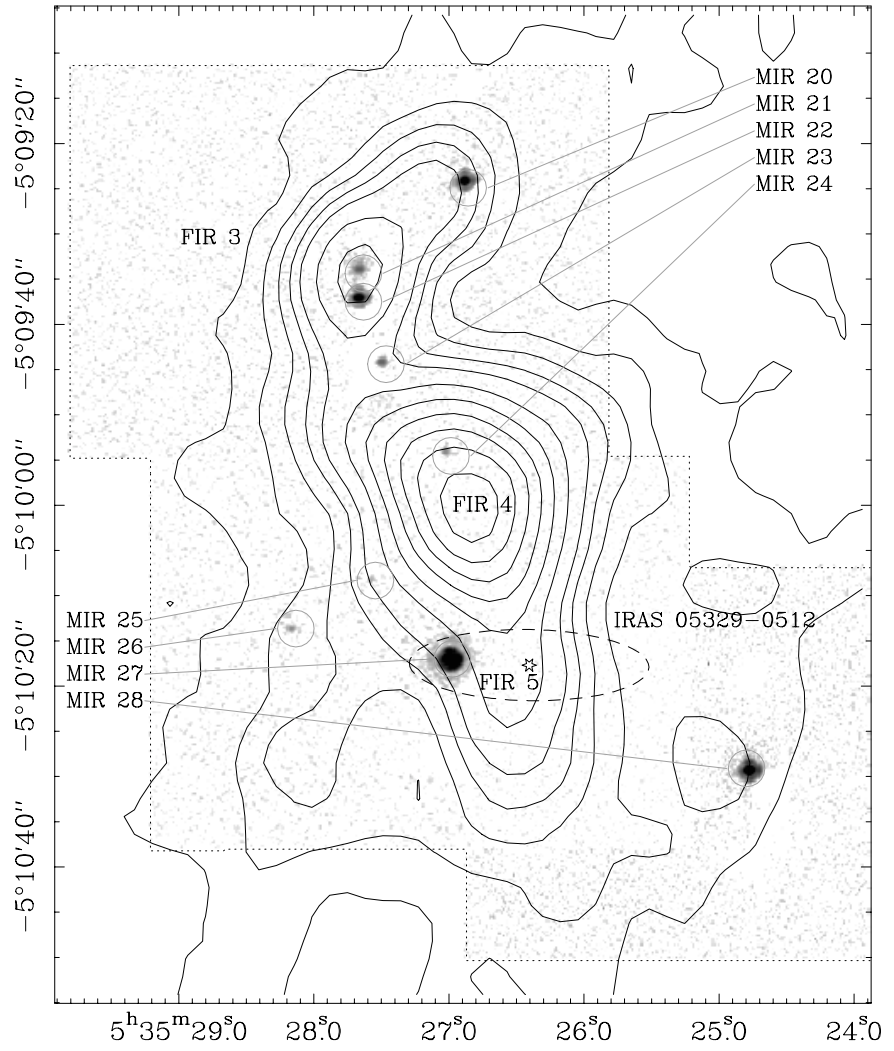

Fig. 11. Superposition of the MIR and $\mathrm{mm}$ data of the region around OMC 2 FIR 4 (MIR 20-28). The grey-scale picture is the TIMMI 2 image showing 9 sources. The contours are taken from the millimetre map of Chini et al. (1997).

display an excess of $\Delta K<1$ while its spectral index $\alpha$ points towards Class I.

FIR 3 contains a binary source (MIR 21/22) with a projected distance of about $1400 \mathrm{AU}$. It was originally discovered as IRS 4 by Gatley et al. (1974) and does not display any significant NIR excess. Its spectral index $\alpha$ puts it among the Class II objects although this classification might be contaminated by the high visual extinction of $A_{V} \sim 22$ mag.

FIR 4 is in close neighbourhood to MIR 24 which is a Class I source with a tiny $K$ excess.

FIR 5 is associated with an IRAS source and close to MIR 27. Its spectral index $\alpha$ suggests Class I; this is corroborated by its $J H K$ colours that imply a small excess of $\Delta K \sim 0.5$.

MIR 28 is a deeply embedded source with a $K$ excess of about 0.5 . It is located in a region of enhanced millimetre emission which, however, has no separate nomenclature (see Fig. 11); it is also of Class I.

\subsubsection{OMC 2 FIR 6}

Figure 12 displays FIR 6 with its four millimetre clumps $a-d$. Only condensation $d$ contains a binary MIR source (MIR 31/32) of which both components are of Class II; their projected distance is $1100 \mathrm{AU}$. Another Class II source (MIR 34) coincides with a faint millimetre clump without nomenclature in the very South-Western part of Fig. 12. 


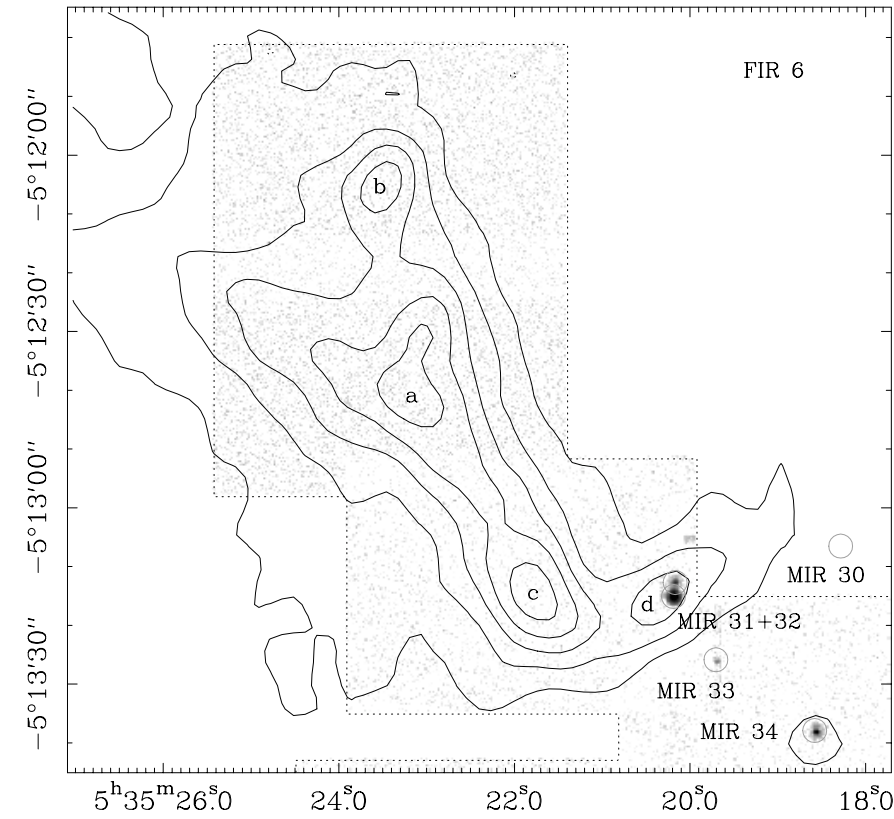

Fig. 12. Superposition of the MIR and $\mathrm{mm}$ data of the region around OMC 2 FIR 6 (MIR 30-34). The grey-scale picture is the TIMMI 2 image showing 5 sources. The position of MIR 30 was determined from the negative signal of the off-position. The contours are taken from the millimetre map of Chini et al. (1997).

\subsubsection{OMC 2 MIR 35-45}

The new Southern extension of the millimetre map by Chini et al. (1997) (see Fig. 2) is enlarged in Fig. 13; it does not have any nomenclatures. There is a rather strong millimetre peak in the very South which coincides with the Class I source MIR 42. Another fainter millimetre clump contains the binary source MIR 39/40 with a projected separation of 380 AU. A similar close binary (MIR 43/44) of Class II and a separation of $420 \mathrm{AU}$ lies far off the millimetre emission ridge and at the Eastern edge of Fig. 13.

\subsection{Global properties of the region}

Here we summarise the major properties of the extended dust emission and the stellar association in OMC 2/3 as they emerge from the present study. Our new millimetre observations with SIMBA plus the Southern extension of our imaging at IRAM reveals faint extended emission filaments and enlarges the number of bona-fide dust condensations to 28. A comparison between the extended MIR emission as obtained from the MSX survey and the millimetre emission shows that the dust emission filaments seen by SIMBA in the Northern (OMC 3) region appear as absorbing infrared dark filaments at the MSX wavebands, indicating the presence of cool and dense dust. Using the method described by Siebenmorgen \& Krügel (2000), we find optical depths at $8.3 \mu \mathrm{m}$ that correspond to visual extinctions of up to 200 mag in the densest regions of OMC 3 . However, the locations of the largest optical depths are not necessarily correlated with the strength of the millimetre emission. In numerous cases, they lie well away from the millimetre peaks. In contrast to that, we find that millimetre emission regions farther to the

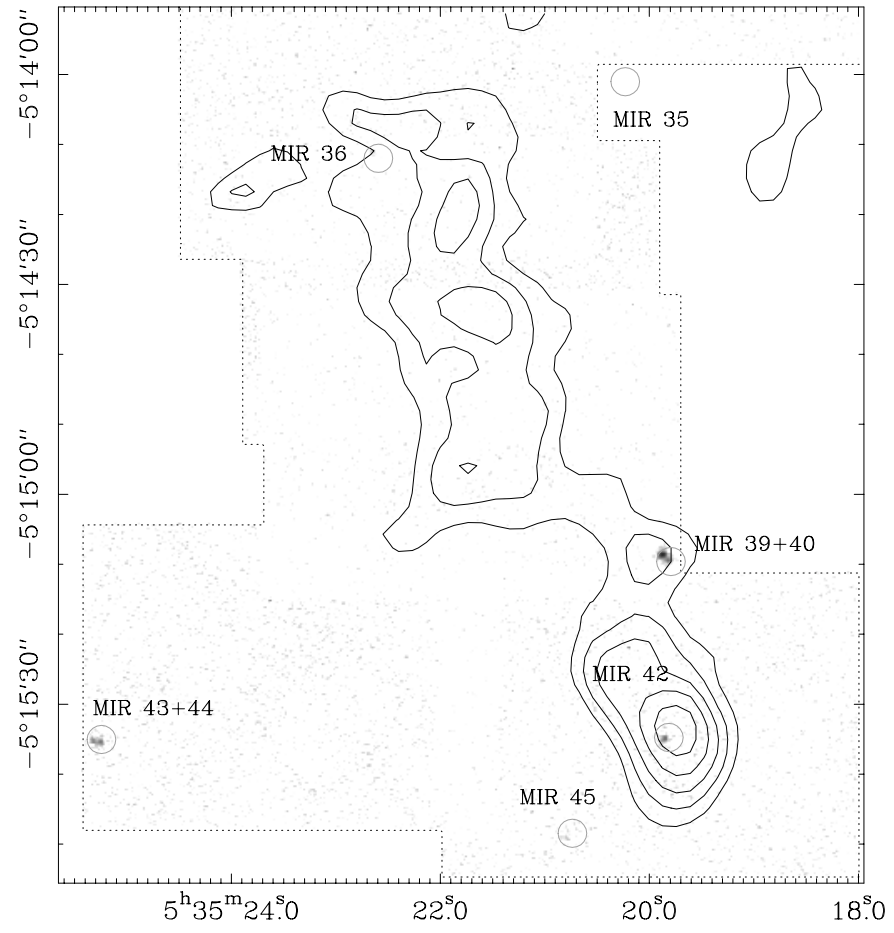

Fig. 13. Superposition of the MIR and $\mathrm{mm}$ data of the region around OMC 2 MIR 35-45. The grey-scale picture is the TIMMI 2 image showing 8 sources. The position of MIR 35 was determined from the negative signal of the off-position. MIR 37 and 38 only have upper limits. The contours are taken from the Southern extension in Fig. 2.

South like e.g. OMC 1, are dominated by the radiation at MIR wavelengths, suggesting considerably higher dust temperatures and lower densities. Free-free emission might also contribute to the mm detections that are located within the $\mathrm{H}$ II regions of the Orion nebula (OMC 1).

Our MIR survey along the dust filaments of OMC $2 / 3$ has revealed 45 sources. Twelve of them belong to binary systems with projected separations between 380 and 1400 AU. These sources can also be identified in the 2MASS survey where they are, however, unresolved. Their combined NIR colours indicate that five systems consist of early spectral types (probably B) while one seems to have later type stars (K). Interestingly, all binaries are associated with millimetre emission which probably arises from circumstellar discs. As mentioned above, in the case of MIR 5/6 the discs are to be seen directly at $10.4 \mu \mathrm{m}$. In OMC 2, the associated millimetre emission decreases from North $(676 \mathrm{mJy})$ to South $(48 \mathrm{mJy})$ maybe suggesting a proceeding disruption of circumstellar discs when approaching the adjacent Orion nebula.

Concerning the evolutionary stages of the objects there is no quantitative relation between the strength of the millimetre emission and the evolutionary stage of the objects as derived from the spectral index $\alpha$ between $K$ and $N$. At most there is a slight statistical bias in the sense that two thirds of the Class I sources are associated with millimetre clumps while only one third of the Class II sources do show significant millimetre emission. The distribution of $J$ luminosities shows that the fraction of high luminosity early type stars is significantly higher in OMC 2 compared to OMC 3. On an even larger scale, 
the luminosities and thus the masses of stars in Orion A seem to decrease from South (OMC 1) to North (OMC 3).

Acknowledgements. We whish to thank the TIMMI 2 team and the telescope team of the ESO $3.6 \mathrm{~m}$ for their excellent support during this first observing run with the new instrument. It is a pleasure for R.C. to thank N. Ageorges for her help and assistance with the observations and $\mathrm{R}$. Siebenmorgen for helpful discussions concerning the calibration. We thank H. Zinnecker for his valuable ideas. Likewise, we want to thank the referee E. Bica for his helpful remarks.

This publication made use of data products from the Two Micron All Sky Survey, which is a joint project of the University of Massachusetts and the Infrared Processing and Analysis Center/California Institute of Technology, funded by the National Aeronautics and Space Administration and the National Science Foundation. This research made use of data products from the Midcourse Space Experiment. Processing of the data was funded by the Ballistic Missile Defense Organization with additional support from NASA Office of Space Science. This research has also made use of the NASA/IPAC Infrared Science Archive, which is operated by the Jet Propulsion Laboratory, California Institute of Technology, under contract with the National Aeronautics and Space Administration.

\section{References}

Ali, B., \& DePoy, D. L. 1995, AJ, 109, 709 (AD)

André, P., Ward-Thompson, D., \& Barsony, M. 1993, ApJ, 406, 122

Bally, J., Langer, W. D., Stark, A. A., \& Wilson, R. W. 1987, ApJ, 312, L45

Carpenter, J. M., Hillenbrand, L. A., \& Skrutskie, M. F. 2001, AJ, 121, 3160 (CHS)

Castets, A., \& Langer, W. D. 1995, A\&A, 294, 835

Chini, R., Reipurth, B., Ward-Thompson, D., et al. 1997, ApJ, 474, L135
Dietzsch, E., \& Reimann, H. G. 1998, SPIE, 2482, 151

Ducati, J. R., Bevilacqua, C. M., Rembold, S. B., \& Ribeiro, D. 2001, ApJ, 588, 309

Gatley, I., Becklin, E. E., Mattews, K., et al. 1974, ApJ, 191, L121

Hillenbrand, L. A., Strom, S. E., Calvet, N., et al. 1998, AJ, 116, 1816

Johnson, J. J., Gehrz, R. D., Jones, T. J., Hackwell, J. A., \& Grasdalen, G. L. 1990, AJ, 100, 518

Johnstone, D., \& Bally, J. 1999, ApJ, 510, L29

Jones, T. J., Mergen, J., Odewahn, S., et al. 1994, AJ, 107, 2120

Kutner, M. L., Tucker, K. D., Chin, G., \& Thaddeus, P. 1977, ApJ, 215,521

Lis, D. C., Serabyn, E., Keene, J., et al. 1998, ApJ, 509, 299

McCaughrean, M. J., \& O’Dell, C. R. 1996, AJ, 111, 1977

Meyer, M. R., Calvet, N., \& Hillenbrand, L. 1997, AJ, 114, 288

Mezger, P. G., Wink, J. E., \& Zylka, R. 1990, A\&A, 228, 95

Nyman, L.-Å., Lerner, M., Nielbock, M., et al. 2001, Msngr, 106, 40 O’Dell, C. R., Wen, Z., \& Hu, X. 1993, ApJ, 410, 696

Rayner, J., McLean, I., McCaughrean, M., \& Aspin, C. 1989, MNRAS, 241, 469

Reimann, H. G., Weinert, U., \& Wagner, S. 1998, SPIE, 3354, 865

Reipurth, B., Rodríguez, L. F., \& Chini, R. 1999, A\&A, 352, L83

Schmidt-Kaler, T. 1982, in Landolt-Börnstein, ed. K. Schaifers, \& H. H. Voigt, vol. VI/2b, Chpt. 4.1.2, 15

SEST operating manual 2002, The SEST Handbook, version 4.3, Chpts. 6 and 8

Siebenmorgen, R., \& Krügel 2000, A\&A, 364, 625

Tatematsu, K., Umemoto, T., Kameya, O., et al. 1993, ApJ, 404, 643

Tsuboi, Y., Koyama, K., Hamaguchi, K., et al. 2001, ApJ, 554, 734

Tsujimoto, M., Koama, K., Tsuboi, Y., et al. 2002, ApJ, 573, 270

Yu, K. C., Bally, J., \& Devine, D. 1997, ApJ, 485, L45

Wainscoat, R. J., \& Cowie, L. L. 1992, AJ, 103, 332

Williams, J. P., Plambeck, R. L., \& Heyer, M. H. 2003, ApJ, 591, 1025 